\title{
Numerical Simulation of Entropy Growth for a Nonlinear Evolutionary Model of Random Markets
}

\author{
Mahdi Keshtkar, ${ }^{1}$ Hamidreza Navidi, ${ }^{1}$ and Elyas Shivanian ${ }^{2}$ \\ ${ }^{1}$ Department of Applied Mathematics, Shahed University, P.O. Box 18155/159, Tehran, Iran \\ ${ }^{2}$ Department of Mathematics, Imam Khomeini International University, Qazvin 34149-16818, Iran \\ Correspondence should be addressed to Hamidreza Navidi; navidi@shahed.ac.ir
}

Received 15 June 2016; Accepted 10 October 2016

Academic Editor: Remi Léandre

Copyright (C) 2016 Mahdi Keshtkar et al. This is an open access article distributed under the Creative Commons Attribution License, which permits unrestricted use, distribution, and reproduction in any medium, provided the original work is properly cited.

\begin{abstract}
In this communication, the generalized continuous economic model for random markets is revisited. In this model for random markets, agents trade by pairs and exchange their money in a random and conservative way. They display the exponential wealth distribution as asymptotic equilibrium, independently of the effectiveness of the transactions and of the limitation of the total wealth. In the current work, entropy of mentioned model is defined and then some theorems on entropy growth of this evolutionary problem are given. Furthermore, the entropy increasing by simulation on some numerical examples is verified.
\end{abstract}

\section{Introduction}

In the last years, it has been reported $[1,2]$ that, in western societies, around $95 \%$ of the population, the middle and lower economic classes of society, arrange their incomes in an exponential wealth distribution. The incomes of the rest of the population, around $5 \%$ of individuals, fit a power law distribution [3]. A kind of models considering the randomness associated to markets is the gas-like models [4]. These random models interpret economic exchanges of money between agents similarly to collisions in a gas where particles share their energy [5]. In this communication, the increasing of the entropy when these systems evolve toward the asymptotic equilibrium is checked. This is associated with the existence of an $\mathrm{H}$-theorem for all these economic models $[6,7]$. Exponential distribution is ubiquitous in the framework of multiagent systems. Usually, it appears as an equilibrium state in the asymptotic time evolution of statistical systems. It has been explained from very different perspectives. In statistical physics, it is obtained from the principle of maximum entropy [8]. In the same context, it can also be derived without any consideration about information theory, only from geometrical arguments under the hypothesis of equiprobability in phase space [9]. Also, several multiagent economic models based on mappings, with random, deterministic, or chaotic interactions, can give rise to the asymptotic appearance of the exponential wealth distribution [10-13]. Concretely, the continuous economic model for random markets is given by

$$
T y(x)=\iint_{u+v \geq x} \frac{y(u) y(v)}{u+v} d u d v .
$$

It is found that the exponential distribution is a stable fixed point of this type of system. In this work, first entropy of mentioned model is defined and then we give some theorems on entropy growth of this evolutionary problem in the next section. Moreover, we illustrate the entropy increasing by simulation on some numerical examples in Section 3. Finally, some concluding remarks are given in Section 4.

\section{Entropy Growth}

Definition 1. We define space $S$ as follows:

$$
\begin{aligned}
S & =\left\{p(x): p(x) \in L_{1}^{+}[0,+\infty),\|p(x)\|\right. \\
& \left.=1, \int_{0}^{+\infty} x p(x) d x=\frac{1}{\alpha}\right\} .
\end{aligned}
$$

Definition 2. For each $p(x) \in S$, we define $H(p)=$ $-\int_{0}^{+\infty} p(x) \log [p(x)] d x$ as entropy of $p(x)$. 
Definition 3. Assume $y(x) \in S$; we introduce a sequence of real numbers as

$$
H_{n}(y)=-\int_{0}^{+\infty} T^{n} y(x) \log \left[T^{n} y(x)\right] d x,
$$

where $T y(x)=\iint_{u+v \geq x}(y(u) y(v) /(u+v)) d u d v$.

Lemma 4. Suppose that $p(x) \in L_{1}^{+}[0,+\infty)$ is decreasing function; then there exists $\beta>0$, so that $\forall x \in[\beta,+\infty)$ : $p(x) \ll|1 / \log [p(x)]|$.

Proof. It is clearly a consequence of this fact that for each $\varepsilon>$ 0 there exists $\delta>0$ so that $\forall 0<x<\delta:|x \log x|<\varepsilon$. Now from this point that $p(x)$ is decreasing, function $p(x)$ tends to zero. Therefore, there exists surely a large number as $\beta$ so that we have $p(x) \ll|1 / \log [p(x)]|$ in the interval $[\beta,+\infty)$.

Theorem 5. Entropy function $H: S \rightarrow \mathscr{R}$ is bounded. In other words, there exist $M$ so that $\forall y(x) \in S:|H(y)|<M$.

Proof. Suppose that $y(x) \in S$; then it is clear that there exists $\varphi \in \mathscr{R}^{+}$so that $y(x)$ is decreasing function in the interval $[\varphi,+\infty)$, because in the case of the fact that function $y(x)$ is not decreasing we have $\int_{\varphi}^{+\infty} y(x) d x=+\infty$ which is contradiction by definition of the set $S$. Now, it is obvious from Lemma 4 for decreasing function $y(x)$ to say that there exist $\beta>\varphi$ and $\gamma, k>1$ and $c>0$ so that $|1 / \log [y(x)]|>$ $1 / c\left(1+x^{k}\right)$ and $y(x)<1 / x^{k+\gamma}$; therefore

$$
\begin{aligned}
& |H(y)|=\left|\int_{0}^{+\infty} y(x) \log [y(x)] d x\right| \\
& =\left|\int_{0}^{\beta} y(x) \log [y(x)] d x+\int_{\beta}^{+\infty} y(x) \log [y(x)] d x\right| \\
& \leq\left|\int_{0}^{\beta} y(x) \log [y(x)] d x\right| \\
& \quad+\left|\int_{\beta}^{+\infty} y(x) \log [y(x)] d x\right| \\
& <M_{1}+\int_{\beta}^{+\infty} y(x)|\log [y(x)]| d x \leq M_{1} \\
& \quad+\int_{\beta}^{+\infty} \frac{c\left(1+x^{k}\right)}{x^{k+\gamma}} d x \\
& =M_{1}+\int_{\beta}^{+\infty} \frac{c}{x^{k+\gamma}} d x+\int_{\beta}^{+\infty} \frac{c}{x^{\gamma}} d x \leq M_{1}+M_{2}+M_{3} \\
& \quad=M_{1},
\end{aligned}
$$

so the proof is completed.

Corollary 6. The sequence of real numbers $H_{n}(y), y(x) \in S$, is bounded sequence.

Proof. It is obviously the consequence of Theorem 5.
Theorem 7. Suppose that $\lim _{n \rightarrow \infty} T^{n} y(x), y(x) \in S$ exists, and then $\lim _{n \rightarrow \infty} H_{n}(y)=1-\log \alpha$.

Proof. We know that $\lim _{n \rightarrow \infty} T^{n} y(x)=\alpha e^{-\alpha x}$. Now, since the function $\log (\cdot)$ is continuous in the interval $(0,+\infty)$, then we have

$$
\begin{aligned}
& \lim _{n \rightarrow \infty} H_{n}(y)=-\lim _{n \rightarrow \infty}\left[\int_{0}^{+\infty} T^{n} y(x) \log \left[T^{n} y(x)\right] d x\right] \\
& =-\int_{0}^{+\infty} \lim _{n \rightarrow \infty}\left[T^{n} y(x)\right] \log \left[\lim _{n \rightarrow \infty}\left[T^{n} y(x)\right]\right] d x \\
& =-\int_{0}^{+\infty} \alpha e^{-\alpha x} \log \left[\alpha e^{-\alpha x}\right] d x \\
& =-\int_{0}^{+\infty} \alpha e^{-\alpha x} \log [\alpha] d x+\int_{0}^{+\infty} \alpha^{2} x e^{-\alpha x} d x \\
& =-\log [\alpha]+1 .
\end{aligned}
$$

Theorem 8. Suppose that $H_{n}(y), y(x) \in S$, is increasing sequence; then the entropy of $\alpha e^{-\alpha x}$ is the supremum among entropies of all distributions in the set $S$.

Proof. Assume that $H_{n}(y)$ is increasing sequence; on the other hand by Corollary 6 we know that $H_{n}(y)$ is bounded. Therefore there is a limit for sequence $H_{n}(y)$; from Theorem 5 this limit should be $1-\log \alpha$; that is,

$$
\lim _{n \rightarrow \infty} H_{n}(y)=1-\log \alpha=H\left(\alpha e^{-\alpha x}\right) .
$$

Then the entropy of $\alpha e^{-\alpha x}$ is the supremum.

Theorem 9. Suppose that $\lim _{n \rightarrow \infty} T^{n} y(x), y(x) \in S$, exists and the entropy of $\alpha e^{-\alpha x}$ is the supremum among the entropies of all distributions in the set $S$; then $H_{n}(y), y(x) \in S$, is increasing sequence for enough large $n \in \mathbb{N}$.

Proof. Existence of $\lim _{n \rightarrow \infty} T^{n} y(x)$ implies that $\lim _{n \rightarrow \infty} H_{n}(y)=H\left(\alpha e^{-\alpha x}\right)$; then for each $\varepsilon>0$ there exist $N$ such that for all $n>N$ we have $\left|H_{n}(y)-H\left(\alpha e^{-\alpha x}\right)\right|<\varepsilon$. On the other hand we know that $H\left(\alpha e^{-\alpha x}\right)$ is supremum; therefore for all $n>N$ we have

$$
H\left(\alpha e^{-\alpha x}\right)-H_{n+1}(y) \leq H\left(\alpha e^{-\alpha x}\right)-H_{n}(y)<\varepsilon .
$$

Hence, $H_{n}(y) \leq H_{n+1}(y)$ which means $H_{n}(y)$ is increasing sequence.

\section{Numerical Simulations}

Example 1. Consider $p(x)=x e^{-x} \in S$; then $\int_{0}^{+\infty} x p(x) d x=$ 2 and so $\alpha=1 / 2$ and

$$
1-\log (\alpha)=1-\log \left(\frac{1}{2}\right)=1.6931
$$


TABLE 1

\begin{tabular}{lccc}
\hline$p(x)$ & $H_{0}(p)$ & $H_{1}(p)$ & $1-\log (\alpha)$ \\
\hline $6 /(1+2 x)^{4}$ & -0.458426 & -0.430621 & -0.386294 \\
$2 \sqrt{2} / \pi\left(1+x^{4}\right)$ & 0.613654 & 0.642990 & 0.653426 \\
$2 \operatorname{sech}[x] / \pi$ & 1.144729 & 1.150917 & 1.153787 \\
$(1-\tanh [x]) / \log [2]$ & 0.473482 & 0.476254 & 0.477918 \\
$4 / \pi\left(1+x^{2}\right)^{2}$ & 0.531024 & 0.540870 & 0.548417 \\
\hline
\end{tabular}

$H_{n}(p)$ for some primary $n$ have been calculated as follows:

$$
\begin{aligned}
& H_{0}(p)=1.5772 \\
& H_{1}(p)=1.6667 \\
& H_{2}(p)=1.6839 \\
& H_{3}(p)=1.6895 \\
& H_{4}(p)=1.6917 \\
& H_{5}(p)=1.6925 .
\end{aligned}
$$

Example 2. Consider $p(x)=e^{-2 x}+e^{-x} / 2 \in S$; then $\int_{0}^{+\infty} x p(x) d x=3 / 4$ and so $\alpha=4 / 3$ and

$$
1-\log (\alpha)=1-\log \left(\frac{4}{3}\right)=0.712318
$$

$H_{n}(p)$ for some primary $n$ have been calculated as follows:

$$
\begin{aligned}
& H_{0}(p)=0.707208 \\
& H_{1}(p)=0.709950 \\
& H_{2}(p)=0.711228 .
\end{aligned}
$$

Example 3. Consider $p(x)=9(\log [10])^{2} x / 10^{3 x} \in S$; then $\int_{0}^{+\infty} x p(x) d x=2 / 3 \log [10]$ and so $\alpha=3 \log [10] / 2$ and

$$
1-\log (\alpha)=1-\log \left(\frac{3 \log [10]}{2}\right)=-0.239498 .
$$

$H_{n}(p)$ for some primary $n$ have been calculated as follows:

$$
\begin{gathered}
H_{0}(p)=-0.355429 \\
H_{1}(p)-0.265942 \\
H_{2}(p)=-0.248720 .
\end{gathered}
$$

Example 4. In this example we have provided Table 1 of other types of distributions in $S$ and calculated some primary $H_{n}(p)$ and corresponding $1-\log (\alpha)$ for each function as well.

\section{Conclusion}

Different versions of a continuous economic model that takes into account idealistic characteristics of the markets have been reconsidered. In these models, the agents interact by pairs and exchange their money in a random way. The asymptotic steady state of these models is the exponential wealth distribution. The system decays to this final distribution with a monotonic increasing of the entropy taking its maximum value just on the equilibrium.

\section{Competing Interests}

The authors declare that they have no competing interests.

\section{References}

[1] A. Dragulescu and V. M. Yakovenko, "Exponential and powerlaw probability distributions of wealth and income in the United Kingdom and the United States," Physica A: Statistical Mechanics and its Applications, vol. 299, no. 1-2, pp. 213-221, 2001.

[2] B. K. Chakrabarti, A. Chatterjee, A. Chakraborti, and S. Sinha, Econophysics: An Introduction, Willey-VCH Verlag, Berlin, Germany, 2010.

[3] M. Levy and S. Solomon, "New evidence for the power-law distribution of wealth," Physica A: Statistical Mechanics and Its Applications, vol. 242, no. 1-2, pp. 90-94, 1997.

[4] V. M. Yakovenko, "Econophysics, statistical mechanics approach to," in Encyclopedia of Complexity and System Science, R. A. Meyers, Ed., Springer, Berlin, Germany, 2009.

[5] A. Dragulescu and V. M. Yakovenko, "Statistical mechanics of money," European Physical Journal B, vol. 17, no. 4, pp. 723-729, 2000.

[6] E. Shivanian and R. Lopez-Ruiz, "A new model for ideal gases. Decay to the Maxwellian distribution," Physica A. Statistical Mechanics and its Applications, vol. 391, no. 8, pp. 2600-2607, 2012.

[7] S. M. Apenko, "Monotonic entropy growth for a nonlinear model of random exchanges," Physical Review E-Statistical, Nonlinear, and Soft Matter Physics, vol. 87, no. 2, Article ID 024101, 2013.

[8] E. T. Jaynes, "Information theory and statistical mechanics," Physical Review E, vol. 106, pp. 620-630, 1957.

[9] R. López-Ruiz, J. Sañudo, and X. Calbet, "A geometrical derivation of the Boltzmann factor," American Journal of Physics, vol. 76, pp. 780-781, 2008.

[10] A. Dragulescu and V. M. Yakovenko, "Evidence for the exponential distribution of income in the USA," European Physical Journal B, vol. 20, no. 4, pp. 585-589, 2001.

[11] J. R. Sánchez, J. González-Estévez, R. López-Ruiz, and M. G. Cosenza, "A model of coupled maps for economic dynamics," The European Physical Journal Special Topics, vol. 143, no. 1, pp. 241-243, 2007.

[12] C. Pellicer-Lostao and R. López-Ruiz, "A chaotic gas-like model for trading markets," Journal of Computational Science, vol. 1, no. 1, pp. 24-32, 2010.

[13] R. López-Ruiz, J. L. López, and X. Calbet, "Exponential wealth distribution: a new approach from functional iteration theory. ESAIM," in Proceedings of the ECIT-2010 Conference, vol. 36, pp. 183-190, April 2012. 


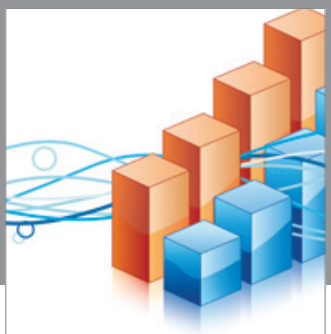

Advances in

Operations Research

vatem alat4

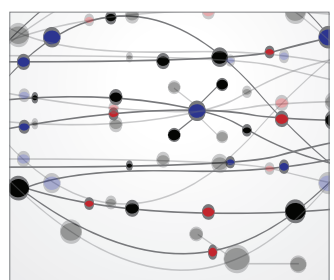

\section{The Scientific} World Journal
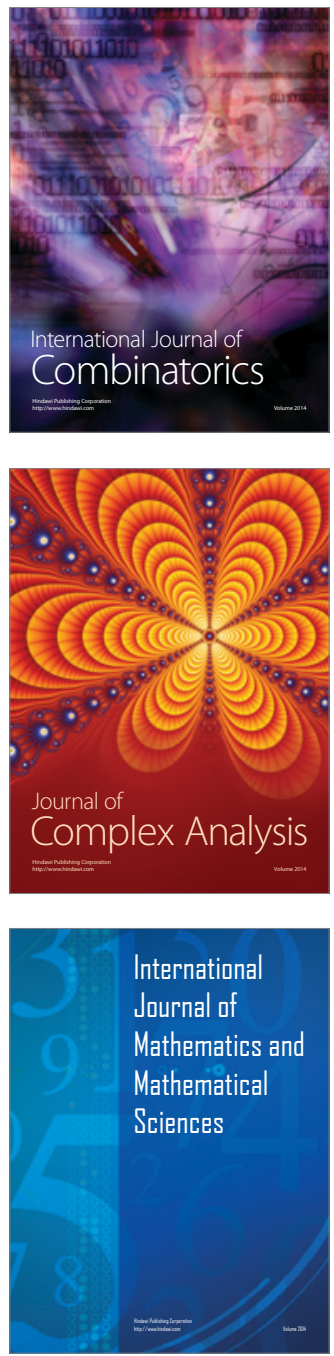
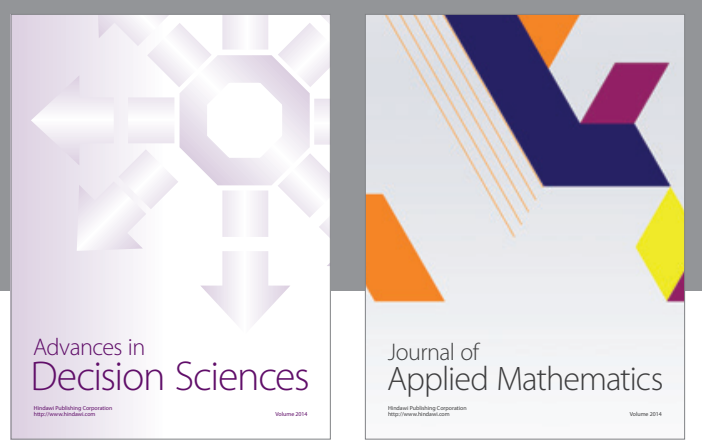

Algebra

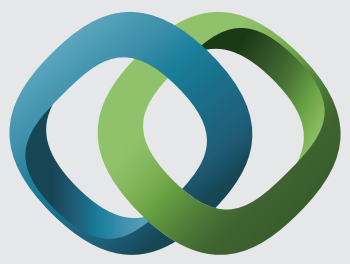

\section{Hindawi}

Submit your manuscripts at

http://www.hindawi.com
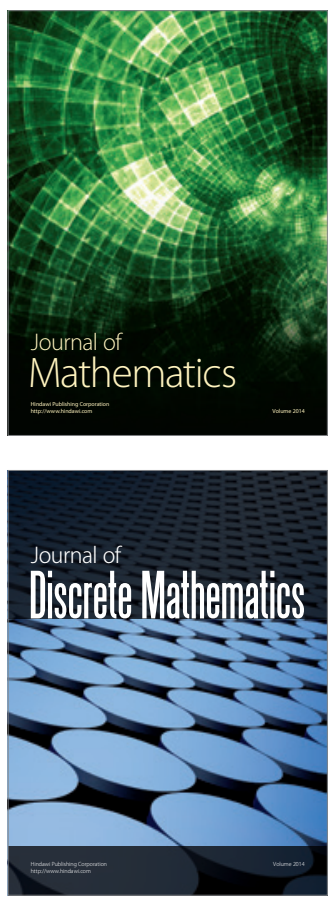

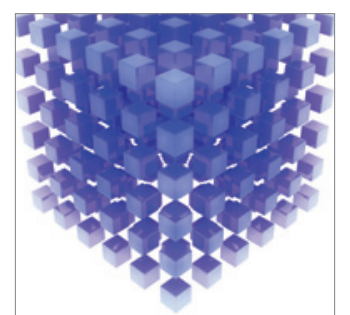

Mathematical Problems in Engineering
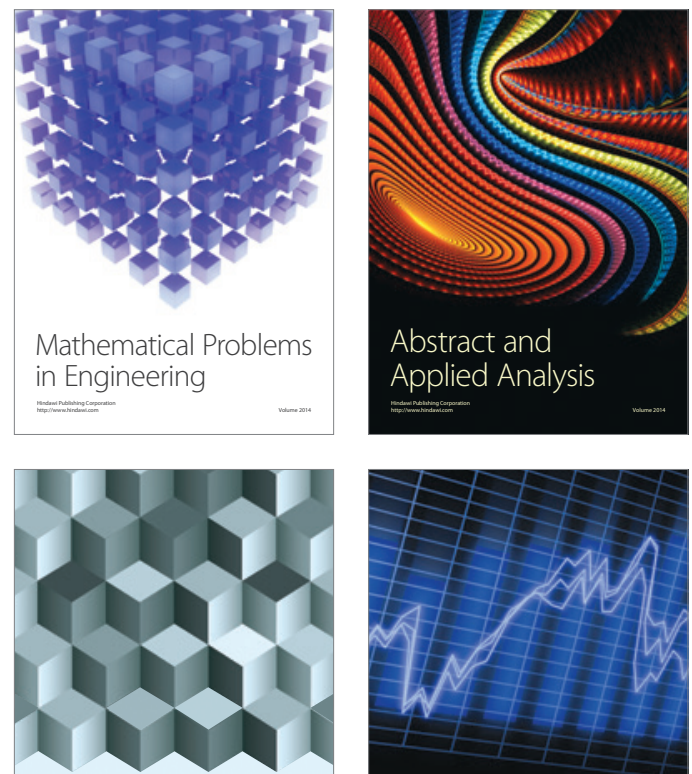

Journal of

Function Spaces

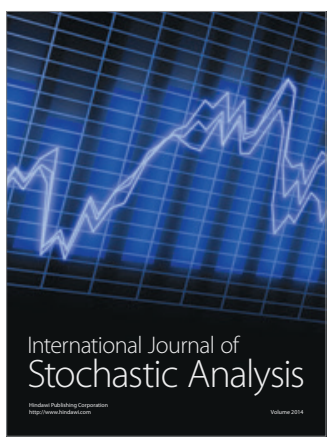

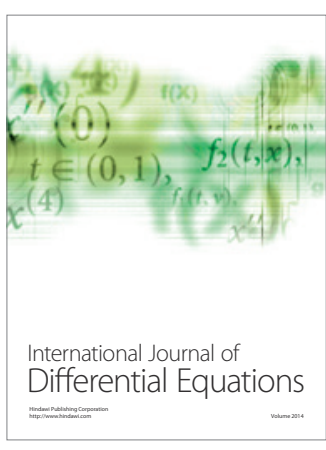
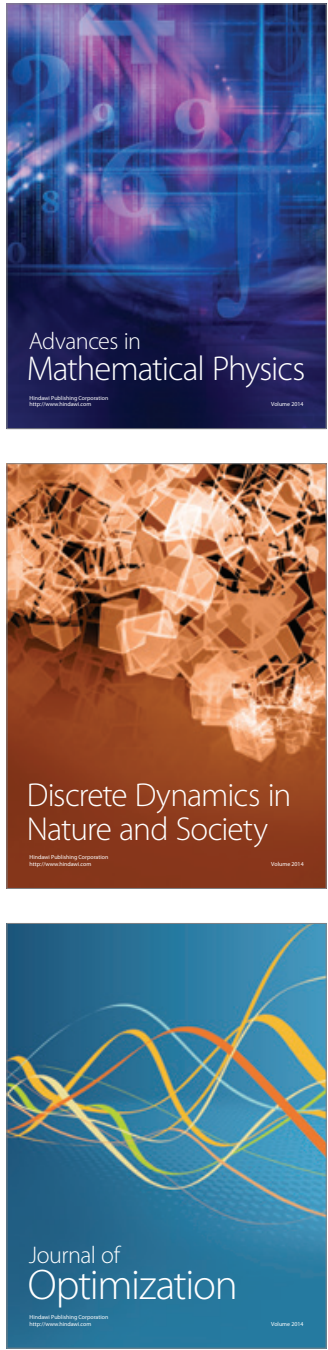